\title{
RESEARCH
}

Open Access

\section{Novel gait training alters functional brain connectivity during walking in chronic stroke patients: a randomized controlled pilot trial}

I-Hsuan Chen ${ }^{1}$, Yea-Ru Yang ${ }^{2}$, Chia-Feng Lu ${ }^{3}$ and Ray-Yau Wang ${ }^{2^{*}}$

\begin{abstract}
Background: A recent study has demonstrated that a turning-based treadmill program yields greater improvements in gait speed and temporal symmetry than regular treadmill training in chronic stroke patients. However, it remains unknown how this novel and challenging gait training shapes the cortico-cortical network and cortico-spinal network during walking in chronic stroke patients. The purpose of this study was to examine how a novel type of gait training, which is an unfamiliar but effective task for people with chronic stroke, enhances brain reorganization.
\end{abstract}

Methods: Subjects in the experimental and control groups received 30 min of turning-based treadmill training and regular treadmill training, respectively. Cortico-cortical connectivity and cortico-muscular connectivity during walking and gait performance were assessed before and after completing the 12-session training.

Results: Eighteen subjects ( $n=9$ per group) with a mean age of $52.5 \pm 9.7$ years and an overground walking speed of $0.61 \pm 0.26 \mathrm{~m} / \mathrm{s}$ consented and participated in this study. There were significant group by time interactions for gait speed, temporal gait symmetry, and cortico-cortical connectivity as well as cortico-muscular connectivity in walk-related frequency $(24-40 \mathrm{~Hz})$ over the frontal-central-parietal areas. Compared with the regular treadmill training, the turning-based treadmill training resulted in greater improvements in these measures. Moreover, the increases in cortico-cortical connectivity and cortico-muscular connectivity while walking were associated with improvements in temporal gait symmetry.

Conclusions: Our findings suggest this novel turning-based treadmill training is effective for enhancing brain functional reorganization underlying cortico-cortical and corticomuscular mechanisms and thus may result in gait improvement in people with chronic stroke.

Trial registration: ACTRN12617000190303. Registered 3 February 2017, retrospectively registered.

Keywords: Stroke, EEG, EMG, Gait, Brain connectivity, Turning

\footnotetext{
* Correspondence: rywang@ym.edu.tw

${ }^{2}$ Department of Physical Therapy and Assistive Technology, National

Yang-Ming University, 155, Sec 2, Li Nong St., Shih-Pai, Taipei 112, Taiwan

Full list of author information is available at the end of the article
}

(c) The Author(s). 2019 Open Access This article is distributed under the terms of the Creative Commons Attribution 4.0 International License (http://creativecommons.org/licenses/by/4.0/), which permits unrestricted use, distribution, and reproduction in any medium, provided you give appropriate credit to the original author(s) and the source, provide a link to the Creative Commons license, and indicate if changes were made. The Creative Commons Public Domain Dedication waiver (http://creativecommons.org/publicdomain/zero/1.0/) applies to the data made available in this article, unless otherwise stated. 


\section{Introduction}

A recent study suggested that chronic stroke patients maintain the capacity to increase synchronization of neural activity between different brain regions as measured by EEG connectivity. These changes of functional connectivity in the motor cortex through neurofeedback correlate with improvements in motor performance [1]. Previously, we demonstrated that a novel specific training, the turning-based treadmill program, yielded greater improvements in gait speed and temporal symmetry than regular treadmill training for people with chronic stroke [2]. We presumed the turning-based treadmill training, which is a challenging and unfamiliar training task for chronic stroke patients, may facilitate brain reorganization and behavioral recovery [3]. Thus, we sought to understand how such novel gait training promotes brain reorganization in this study.

An EEG-based method has the advantage of real-time recording during walking due to the relative ease of data acquisition. As indicated by the authors of the first study to use an EEG signal recorded during walking, the power increases within numerous frequency bands $(3-150 \mathrm{~Hz})$ in the sensorimotor cortex and is more pronounced during the end of the stance phase of walking [4]. Source localization EEG analysis revealed the importance of the primary somatosensory, somatosensory association, primary motor and cingulate cortex in gait control [5]. Focal lesions due to stroke may not only affect the functional connectivity of cortical areas [6] but also impede the neural transmission of descending motor pathways [7]. Based on spectral analysis, the direct relationship of cortical activities with peripheral movements is still unknown. Accordingly, an analysis of EEG-EMG coherence recorded during treadmill walking was done by Petersen et al. [8], who demonstrated that cortical activity in the primary motor cortex within the gamma band $(24-40 \mathrm{~Hz})$ was transmitted via the corticospinal tract to the leg muscles during the swing phase of walking. In addition, a recent study confirmed the strong correlation between kinematic errors of the lower extremities and fronto-centroparietal connectivity during gait training and post-training in healthy subjects [9]. However, it remains unknown how novel and challenging gait training shapes the cortico-cortical network and cortico-spinal network during walking in individuals with chronic stroke. Therefore, the aims of the current study were to explore the effects of the turning-based treadmill training, a novel gait training program, on cortico-cortical connectivity and corticomuscular connectivity and to investigate the relationship between connectivity changes and gait performance in chronic stroke patients.

\section{Methods}

\section{Participants}

Participants with chronic stroke were recruited from medical centers and the surrounding community. Stroke diagnosis, age, gender, stroke type, lesion side, and duration of hemiparesis were obtained from patient interviews and medical charts. To be included in the study, participants with stroke had to satisfy the following criteria: (a) first stroke with unilateral motor deficits at least 6 months prior, (b) ability to walk independently for at least $6 \mathrm{~m}$ with or without the use of stick, quad stick or AFO (to ensure they were able to complete the walk test used in this study), (c) a Brunnstrom stage greater than 3 for the affected lower extremity, and (d) a score of $\geq 24$ on the mini-mental state examination (MMSE). Exclusion criteria were (a) unstable medical conditions (e.g., deep vein thrombosis, aspiration pneumonia, or superimposed sepsis) and (b) history of other diseases known to interfere with participation in such a study (e.g., heart failure, hemi-neglect, or diabetic neuropathy).

\section{Experimental design}

The study was a single-blind, parallel randomized, controlled trial. A research assistant enrolled the participants at the medical centers. An individual unassociated with any other study procedure generated the random allocation sequence and selected one of a set of sealed envelopes to assign participants to either the experimental or control group (block randomization; allocation ratio $=1$ ) before the intervention began. Participants received $30 \mathrm{~min}$ of turning-based treadmill training (experimental group) or regular treadmill training (control group), followed by a 10-min ambulation training program, for 12 sessions over a 4-week period. All training procedures were implemented under a physical therapist's supervision. All outcomes were measured the day before intervention (pre) and the day after completing the intervention (post) by a physical therapist blinded to the group assignment. The study outcomes (see below) included neurophysiological measures during treadmill walking and walking performance on the ground. Participants provided written informed consent for all study procedures, which were approved by the institutional review board of Taipei City Hospital. This trial was registered at https://www.anzctr.org.au/Trial/Registration/TrialReview.aspx?id=372132 (ACTRN12617000190303).

\section{Interventions \\ Turning-based treadmill training}

A rotational treadmill was designed to provide turning-based treadmill training [2]. This treadmill is similar to a regular treadmill except for its circular running motor belt (0.8-m radius), which forces patients to continually turn rather than walk straight. Participants walked on the perimeter of the circular belt as it rotated either clockwise or counterclockwise. Participants were trained in both directions, with the affected leg as the inner limb for $15 \mathrm{~min}$ and then as the outer limb for $15 \mathrm{~min}$ with a 5 -min break in between. The order of walking with the affected limb as the inner or outer leg was alternated in each consecutive session. All participants wore 
an unweighted safety harness to prevent falls. Participants were also allowed to place their hands on the handrail for balance support but were instructed to refrain from holding the handrail. As progressively faster speeds are needed to continue challenging the locomotor abilities of individuals with hemiparesis [10], the treadmill speed, which began at each individual's comfortable turning speed on level ground, was increased by increments of $0.05 \mathrm{~m} / \mathrm{s}$ every $5 \mathrm{~min}$ as tolerated. After completing the turning-based treadmill training, a 10-min ambulation training followed [2].

\section{Regular treadmill training}

The control group received training on a standard treadmill (Biodex, Shirley, NY). Other than the type of treadmill, the training protocol was the same as that described for the experimental group. Training speed was initially set at the individual's comfortable walking speed on level ground and increased by increments of $0.05 \mathrm{~m} / \mathrm{s}$ every $5 \mathrm{~min}$ as tolerated. Participants were trained in two 15-min phases, followed by a 10-min ambulation training as in the experimental group.

\section{Outcome measures}

\section{Functional brain connectivity during walking}

The motor system consists of well-tuned interactions of excitatory and inhibitory mechanisms in the cortical and subcortical areas. Many researchers have used coherence analysis, a method of power spectral cross correlations between spatially separate neural systems, to reflect the functional communication between two systems, i.e., coupling between two brain regions (EEG-EEG coherence) [6], or between cortical commands and consequent muscle activation (EEG-EMG coherence) [7]. However, the conventional coherence methods show linear dependency and may be insufficient to study the nonlinear signals in biological and physical systems, such as data pertaining EEG and EMG [11], especially if the signals are contaminated by noise or the oscillatory frequency band is not carefully defined $[12,13]$.

In the present study, the time-frequency cross mutual information (TFCMI) method was used to solve the problems mentioned above, to calculate the mutual information between two temporal power sequences within a task-specific band between two neural systems $[14,15]$, and thus to examine the treatment-induced brain reorganization during walking. To obtain consistent information, in both the experimental and control groups, subjects walked on the regular treadmill for 5 min while the neurophysiological measures and the functional connectivity of the signals from different scalp locations (EEG-EEG connectivity) and the brain and muscle (EEG-EMG connectivity) were assessed .

EEG, EMG, and footswitch signals during treadmill walking were simultaneously recorded from $\mathrm{Ag} / \mathrm{AgCl}$ electrodes using a 40-channel QuickAmp amplifier (32 EEG channels, 4 bipolar channels for EMG and 4 auxiliary channels for footswitch) and Brain Vision Recorder software (Brain Products, Gilching, Germany).

Using the international 10-20 system, 32 EEG electrodes located over the frontal-parietal areas were used (F5, F3, F1, Fz, F2, F4, F6, FC5, FC3, FC1, FCZ, FC2, FC4, FC6, C5, C3, C1, CZ, C2, C4, C6, CP5, CP3, CP1, CPZ, $\mathrm{CP} 2, \mathrm{CP} 4, \mathrm{CP} 6, \mathrm{P} 1, \mathrm{Pz}, \mathrm{P} 2, \mathrm{POz})$. Electrodes were recorded against an average reference calculated by the amplifier hardware. Horizontal and vertical electrooculography (HEOG and VEOG) signals were also recorded. EEG and EOG signals were amplified with a bandpass of $0.16-50 \mathrm{~Hz}$, recorded in DC mode and sampled at 1000 $\mathrm{Hz}$. Electrode impedances were kept below $5 \mathrm{k} \Omega$ [16].

For recording EMG, the skin was shaved and cleaned with alcohol swabs to reduce impedance before applying $11 \mathrm{~mm} \mathrm{Ag-AgCl}$ surface electrodes (Brain Products, Gilching, Germany). Surface electrodes were placed on the motor point of the tibialis anterior (TA) of the affected leg, which is located at $1 / 3$ on the line between the tip of the fibular and the medial malleolus [17]. Electrode impedances were kept below $5 \mathrm{k} \Omega$.

Four footswitches were placed respectively under the heel and the big toe of each leg and taped to the sole of the foot using medical tape to measure the "heel strike" and "toe-off" events of the gait cycle. Signals from footswitches were recorded using the QuickAmp amplifier and Brain Vision Recorder software to synchronize signals from footswitches with those of the EEG and EMG. Significant coupling between EEG and EMG signals using the conventional EEG-EMG coherence method was found approximately $24-40 \mathrm{~Hz}$ during the swing phase of walking in healthy human subjects [8]. Therefore, the target gait event in this study was focused on the swing phase. The swing phase was defined as the time interval between toe-off and the subsequent heel strike of the same foot.

EEG-EEG connectivity and EEG-EMG connectivity were calculated using the TFCMI method. The data recorded within the target gait event was off-line processed as follows. The independent component analysis (ICA) algorithm was first applied to decompose EEG recordings into 15 independent components. Among these independent components, the signals that were highly correlated to the VEOG or HEOG (with correlation coefficients higher than 0.6) were rejected from the reconstruction to eliminate the potential contamination from eye movement during recording. The EEG/EMG recordings were then segmented into epochs measuring from 200 sample points before the onset of the "toe-off" to 300 sample points after the onset of the "toe-off". The epochs with a signal fluctuation more than a standard deviation from the mean signal value were then discarded to remove potential motion 
artifact and ensure signal stability. The stable EEG signal over time within the same patient was obtained through ICA-based artifact rejection. The Butterworth zero-phase filters with pass band from 1 to $50 \mathrm{~Hz}$ were applied. Each window of filtered EEG/EMG data across channels was transformed into the time-frequency domain using the Morlet wavelet transformation to obtain temporal spectral (power) map [18]. The corresponding Morlet wavelet transformation was given by time-frequency maps encompassing the alpha $(8-12 \mathrm{~Hz})$ and gamma $(24-40$ $\mathrm{Hz}$ ) activities that were created separately within each window. The power across the selected frequency bands in each channel was averaged to produce power curves of the target bands, i.e., alpha and gamma bands in this study. The temporal series of the averaged power signals of the target bands in each channel were used to compute the cross mutual information between any two channels in each window. A full mathematical description of TFCMI has been published previously [14]. The TFCMI value, which is an index of functional connectivity that estimates the relationship between two channels on their averaged power changes over the target band, was calculated based on the entropy and joint entropy. The TFCMI between the $\mathrm{i}^{\text {th }}$ and $\mathrm{j}^{\text {th }}$ channels were calculated as follows:

$$
\begin{aligned}
\operatorname{TFCMI}\left(F_{i}, F_{j}\right) & =H\left(F_{i}\right)+H\left(F_{j}\right)-\left(F_{i}, F_{j}\right) \\
& =\sum_{b=1}^{50} p\left(F_{i, b}, F_{j, b}\right) \ln \frac{p\left(F_{i, b}, F_{j, b}\right)}{p\left(F_{i, b}\right) p\left(F_{j, b}\right)}
\end{aligned}
$$

$\mathrm{H}$ : the entropy; $\mathrm{F}$ : the averaged power signals at the channel; P: the probability density function (pdf); b: the index of sampling bins used to construct the approximated pdf.

The cortical maps of EEG-EMG connectivity were presented in individual channels (32 channels). Considering pairwise connectivity, a 32-channel network for the EEG-EEG connectivity may produce $(32 \times 32-32) / 2$ possible functional connections, which may be convoluted when visualized. Therefore, the 32 channels were grouped into 13 regions to better visualize the EEG-EEG connection between cortical regions (Table 1).

\section{Gait performance}

The gait speed and symmetry measure independent features. Gait speed provides the overall gait performance and may differentiate levels of disability, while the temporal gait symmetry indicates differences of single support time between two limbs due to the difficulty of bearing weight on the affected leg during the stance phase or in advancing the affected leg during the swing phase $[19,20]$. The speed and temporal asymmetry ratio at a comfortable walking speed were obtained from the GAITRite system (CIR system, Inc., Havertown, PS);
Table 1 The divided cortical regions and their abbreviations

\begin{tabular}{lll}
\hline Abbreviation & Cortical location & Channels \\
\hline IF & Ipsilesional frontal area & F4, F6 \\
MF & Middle frontal area & F1, Fz, F2 \\
CF & Contralesional frontal area & F5, F3 \\
IFC & Ipsilesional frontal-central area & FC4, FC6 \\
MFC & Middle frontal-central area & FC1, FCz, FC2 \\
CFC & Contralesional frontal-central area & FC5, FC3 \\
IC & Ipsilesional central area & C4, C6 \\
MC & Middle central area & C1, Cz, C2 \\
CC & Contralesional central area & C5, C3 \\
ICP & Ipsilesional central-parietal area & CP4, CP6 \\
MCP & Middle central-parietal area & CP1, CPz, CP2 \\
CCP & Contralesional central-parietal area & CP5, CP3 \\
MP & Middle parietal area & P1, Pz, P2, POz \\
\hline
\end{tabular}

these two gait parameters were selected based on the results of our previous study [2]. The concurrent validity [21] and reliability [22] of the GAITRite system for stroke subjects have been established. The GAITRite walkway was $4.75 \mathrm{~m}$ long and $0.9 \mathrm{~m}$ wide, and the pressure-sensitive area was $4.30 \mathrm{~m}$ long and $0.61 \mathrm{~m}$ wide. The contact time and location of each footfall were recorded and the gait parameters were calculated on a laptop with application software. Participants began their trials $1.5 \mathrm{~m}$ before the mat and continued walking for $1.5 \mathrm{~m}$ beyond the end of the mat to eliminate the effect of acceleration and deceleration [22]. Participants were allowed to use their customary ankle-foot orthosis or assistive devices [22]. The assistive device and/or ankle foot orthosis used at the preintervention assessment were also used at the postintervention assessment. Fragmented steps and unrelated marks from assistive devices were removed automatically by the GAITRite software or manually by the investigators [22]. The average of three trials was used for data analysis [22]. The temporal asymmetry ratio was calculated using the following formulas [2]:

$$
\begin{aligned}
& \text { Temporal asymmetry ratio } \\
& =\left|1-\frac{\text { single support time }(\text { affected })}{\text { single support time }(\text { unaffected })}\right|
\end{aligned}
$$

\section{Statistical analysis}

All analyses were performed using the SPSS 17.0 statistical software. Descriptive statistics were generated, and distributions of the variables were expressed as the mean \pm standard deviation. Intergroup differences among baseline characteristics were evaluated using an independent $\mathrm{t}$ test or $\mathrm{x} 2$ analysis. Two-way analysis of 
variance with repeated measures was used to determine the effects of intervention on each dependent variable. Model effects were group (experimental, control), time (pre, post), and their interactions. Post hoc independent $t$ tests between groups were used to examine significant models. The association between neurophysiological measures during treadmill walking with significant intergroup differences and training-related changes in gait performance was performed using a Pearson correlation test. Statistical significance was set at .05 .

\section{Results}

Nine participants in the experimental group and 9 participants in the control group completed the study protocol and had sufficient successful trials of functional and neurophysiological data. No adverse effects were reported or noted. The characteristics and clinical information of participants are shown in Table 2. There were no significant differences in baseline demographic or clinical features between the groups. Similarly, there were no significant group differences in any of the preintervention outcome measures.

\section{Functional brain connectivity during walking}

The results of TFCMI analysis in EEG-EEG connectivity are shown in Figs. 1 and 2. Significant group by time interactions were noted in the gamma band. Post hoc testing showed that the experimental group demonstrated significant increases in the EEG-EEG connectivity in multiple areas compared with those of the control group. The experimental group demonstrated significant pre- and postintervention differences in EEG-EEG connectivity, but the control group did not show significant pre- and postintervention differences. The connectivity increases are primarily distributed in the middle central area (MC-IF, MC-MF, MC-CF, MC-IFC, MC-MFC, MC-CFC, MC-MC, MC-CC, MC-ICP, MC-MP) (Fig. 1a), secondarily in the contralesional frontocentral regions (CFC-CFC, CFC-IC, CFC-MC CFC-CC, CFC-ICP, CFC-MCP, CFC-CCP, CFC-MP) (Fig. 1b), and thirdly in the ipsilesional centroparietal regions (ICP-IFC, ICP-MFC, ICP-CFC, ICP-IC, ICP-MC, ICP-CC) (Fig. 1c). The increase in the interhemisphric connectivity included 4 pairs, IF-CCP, CFC-IC, CFC-ICP, and CC-ICP. However, no significant interactions were observed in the alpha band. Figure 2a depicts the areas (total 28 pairs) exhibiting increases in connectivity.

The results of TFCMI analysis in EEG-EMG connectivity are shown in Figs. 3 and 4. In the gamma band, significant interactions between group and time were observed for EEG-EMG connectivity over the frontal and central areas. Post hoc analysis revealed that the experimental group demonstrated significant increases in EEG-EMG connectivity over middle frontal area (Fz, F2), ipsilesional frontal area (F4, F6), contralesional central area $(\mathrm{C} 3, \mathrm{C} 5)$, and middle central area $(\mathrm{C} 1, \mathrm{Cz}$, C2) after training compared to the control group (Fig. 3a and b). The experimental group demonstrated significant pre- and postintervention differences in the EEG-EMG connectivity, but the control group did not show significant pre- and postintervention differences.

For the alpha band, significant interactions between group and time were observed over the frontal and central areas. Post hoc testing indicated that the experimental group demonstrated significant increases in the EEG-EMG connectivity over the contralesional frontal area (F5, F3), middle frontal area (F1, Fz, F2), ipsilesional frontal area (F4, F6), contralesional central area (C3), middle central area $(\mathrm{C} 1, \mathrm{Cz}, \mathrm{C} 2)$ and ipsilesional central area (C4) compared to the control group (Fig. 4a and b). The experimental group demonstrated significant preand postintervention differences for almost all EEG-EMG connectivity, except for F3, C3, and $\mathrm{Cz}$, but the control group did not show significant pre- and postintervention differences.

Table 2 Baseline demographic and clinical characteristics of participants

\begin{tabular}{llll}
\hline Parameters & Control group $(n=9)$ & Experimental group $(n=9)$ & $P$ \\
\hline Age, $y$ & $50.33 \pm 10.95$ & $54.67 \pm 8.32$ & $9 / 0$ \\
Gender, male/female & $8 / 1$ & $66.33 \pm 8.44$ & 1.000 \\
Body weight, $\mathrm{kg}$ & $68.33 \pm 9.77$ & $168.89 \pm 4.34$ & 0.648 \\
Height, $\mathrm{cm}$ & $168.0 \pm 6.42$ & $3 / 6$ & 0.735 \\
Side of hemiparesis, right/left & $3 / 6$ & $3 / 6$ & 1.000 \\
Type of stroke, hemorrhagic/ischemic & $6 / 3$ & $2.86 \pm 2.40$ & 0.347 \\
Time poststroke, y & $3.08 \pm 2.17$ & $4.3 \pm 0.7$ & 0.839 \\
Brunnstrom stage of lower extremity & $4.5 \pm 0.8$ & $3 / 6$ & 0.644 \\
Walking aids used, yes/no & $5 / 4$ & $8 / 1$ & 0.637 \\
Rehabilitation ongoing, yes/no & $8 / 1$ & & 1.000 \\
\hline
\end{tabular}

Values are the mean \pm standard deviation or frequency 

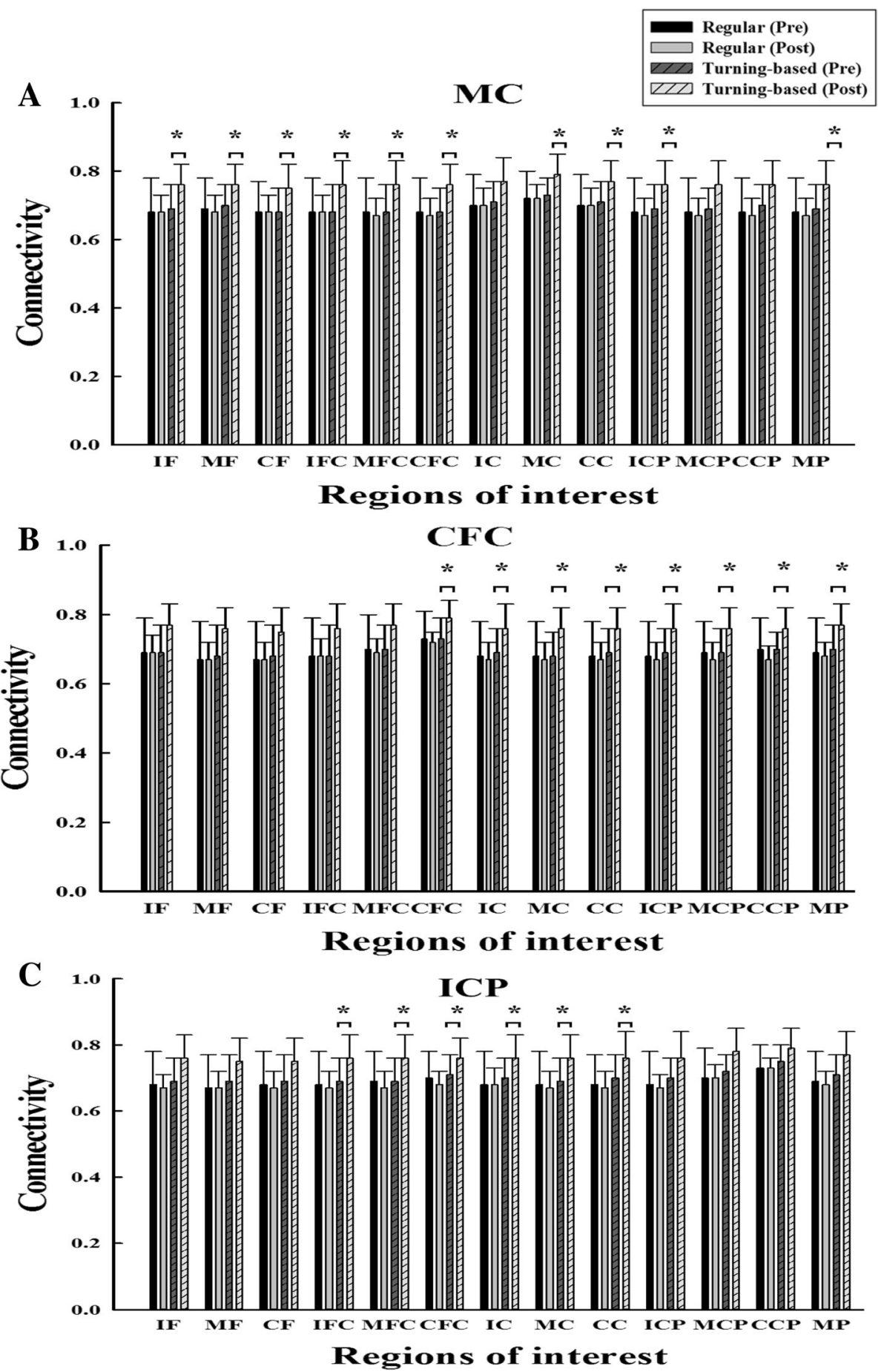

Fig. 1 The mean and standard variations of the EEG-EEG connectivity values in the gamma band are shown for the (a) MC-13 regions, (b) CFC-13 regions, and (c) IPC-13 regions. * denotes a significance level $<0.05$ for intergroup comparisons (control vs. experimental)

\section{Gait performance}

Group by time interactions $(p<0.040)$ were noted for both walking speed and asymmetry ratio (Table 3 ). Compared to the control group, the experimental group demonstrated greater improvements in walking speed $(p=0.037)$ and temporal asymmetry ratio $(p=0.026)$. For the within group comparisons, the experimental group improved significantly from pre- to postintervention for both gait speed $(p=0.011)$ and asymmetry ratio $(p=0.006)$, but the control group did not show significant differences in gait speed and asymmetry ratio. The individual gait details assessed before and after intervention are presented in the Table 4. All 


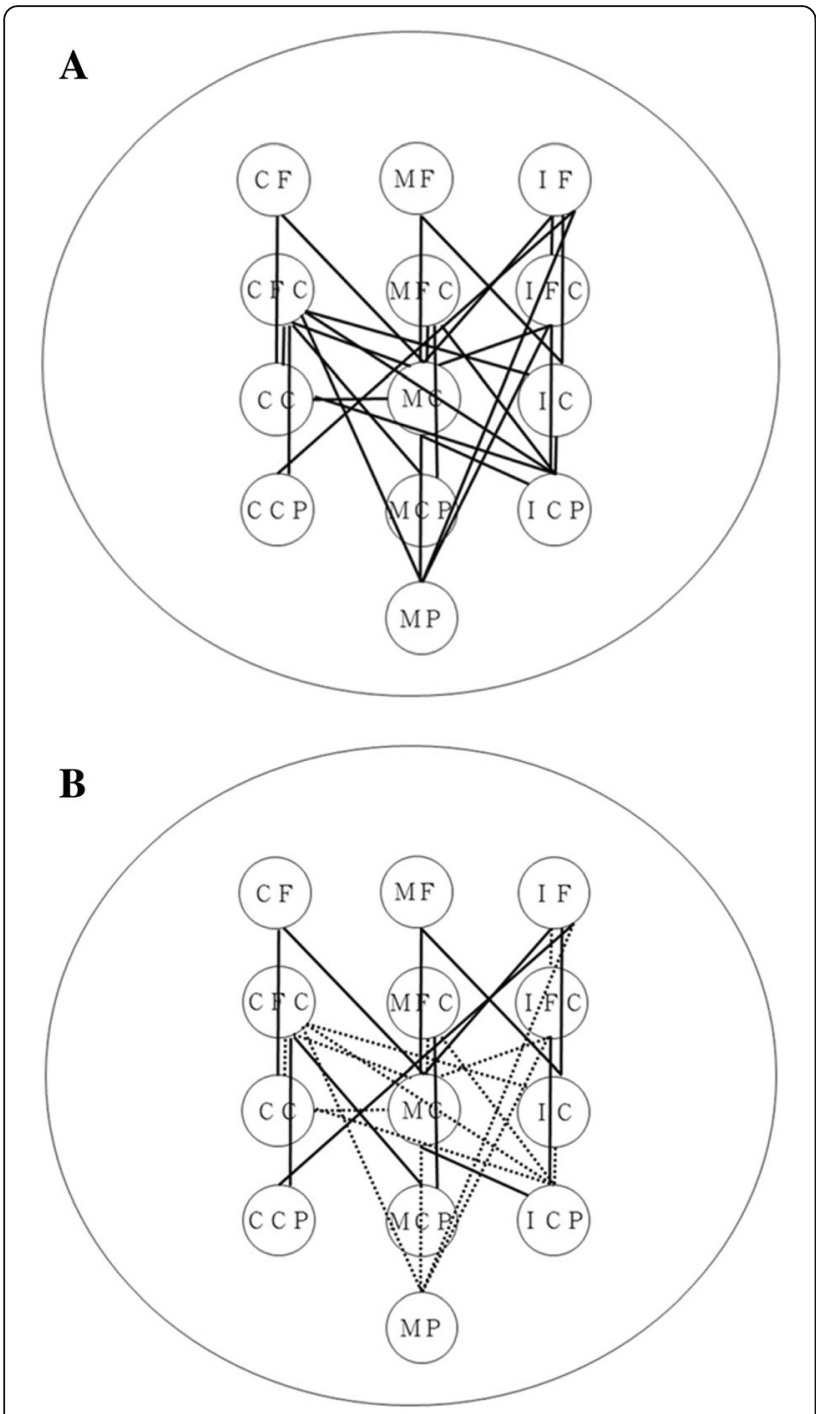

Fig. 2 (a) Functional EEG-EEG connectivity in the gamma band with significant intergroup differences. The signals were recorded during regular treadmill walking. The lines represent the between-region connectivity. (b) Changes in functional EEG-EEG connectivity in the gamma band correlate with the recovery of temporal gait asymmetry. The solid lines represent the changes in the temporal asymmetry ratio that were negatively correlated with the changes in the EEG-EEG pairs. The dotted lines represent the changes in the temporal asymmetry ratio that were not correlated with the changes in the EEG-EEG pairs. For better visualization and interpretation, the left represents the contralesional hemisphere, and the right represents the ipsilesional hemisphere. The abbreviations of the cortical areas are listed in Table 1

participants, except for \#2 in the control group (Table 4), demonstrated the "temporal asymmetry" at the beginning (normative range: $<0.1$ ) [23].

\section{Association between neurophysiological measures and gait recovery}

Correlations between the changes in EEG-EEG pairs exhibiting intergroup differences and gait parameters are shown in Fig. 2b. The temporal asymmetry ratio changes were negatively correlated with the EEG-EEG connectivity changes in the CF-CC, CF-MC, MF-MC, MF-IC, IF-MC, IF-IC, IF-CCP, CFC-CCP, CFC-MCP, MFC-MCP, IFC-ICP, and MC-ICP pairs (ranging from -0.472 to $0.561, p<0.05)$. The changes in gait speed, however, did not correlate significantly to the changes in any EEG-EEG pairs.

Correlations between changes in EEG-EMG pairs exhibiting intergroup differences and gait parameters are shown in Figs. 3c and 4c. For the gamma band, the temporal asymmetry ratio changes were negatively correlated with the EEG-EMG connectivity changes in all pairs (ranging from -0.472 to $-0.653, p<0.05$ ). For the alpha band, the temporal asymmetry ratio changes were negatively correlated with the EEG-EMG connectivity changes over the middle frontal area (Fz, F2), ipsilesional frontal area (F4, F6) and ipsilesional central area (C4) (ranging from -0.516 to $-0.643, p<0.05$ ). The changes in gait speed did not correlate with the changes in any EEG-EMG pairs for the alpha or gamma band.

\section{Discussion}

In this study, we demonstrated that EEG-EEG connectivity and EEG-EMG connectivity during walking can be enhanced more by a novel gait training program that uses a turning-based treadmill instead of a regular treadmill. Moreover, the improvement in gait symmetry, but not the gait speed, correlated with the modulations in the EEG-EEG and EEG-EMG connectivity over frontal-central-parietal areas of the brain.

Restoring walking ability, regardless of speed or symmetry, is an important goal of poststroke rehabilitation, but conventional approaches are not always successful at improving gait asymmetry $[24,25]$. In our previous and present study, we found that changes in walking speed and temporal symmetry were considered clinically significant as well as statistically significant following the turning-based treadmill training [2, 22]. Interlimb asymmetry of the single limb support time stems from less time spent on the affected limb or more time spent on the unaffected limb. Less time on the affected limb may indicate poorer balance control of the affected limb, and more time on the unaffected limb may be due to difficulty advancing the affected leg [23]. During turning-based treadmill training, the outer limb requires relatively greater activation of the ankle dorsiflexors to swing and greater activation of the ankle plantar flexors for propulsion [26]. Alternately, the inner limb increases extensor muscle activity for stance and increases ankle dorsiflexors use for rapid swing [26, 27]. When we reviewed the individual data on the single support time of the unaffected limb and affected limb, we found that normalization of temporal asymmetry in our participants 
A
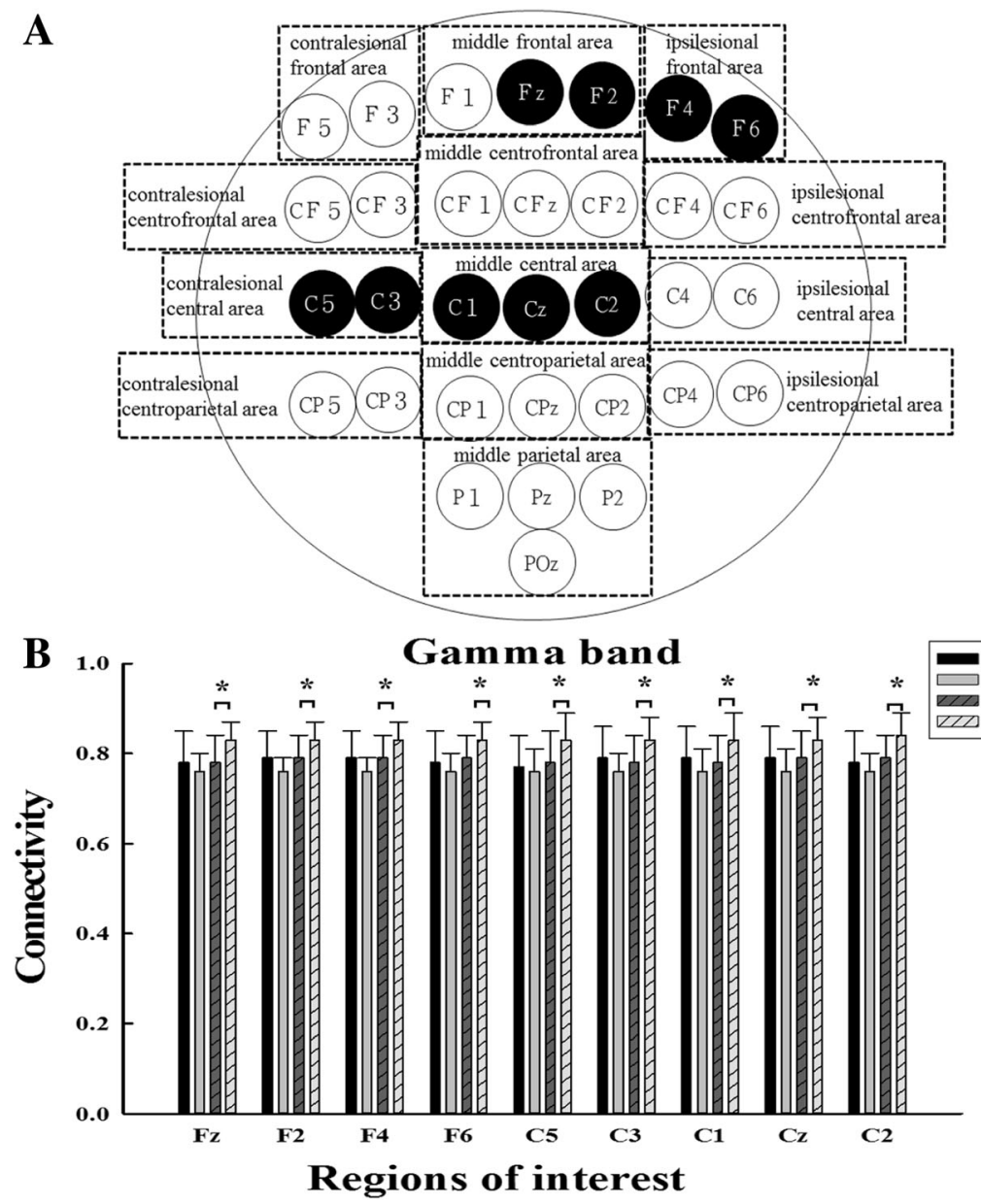

C

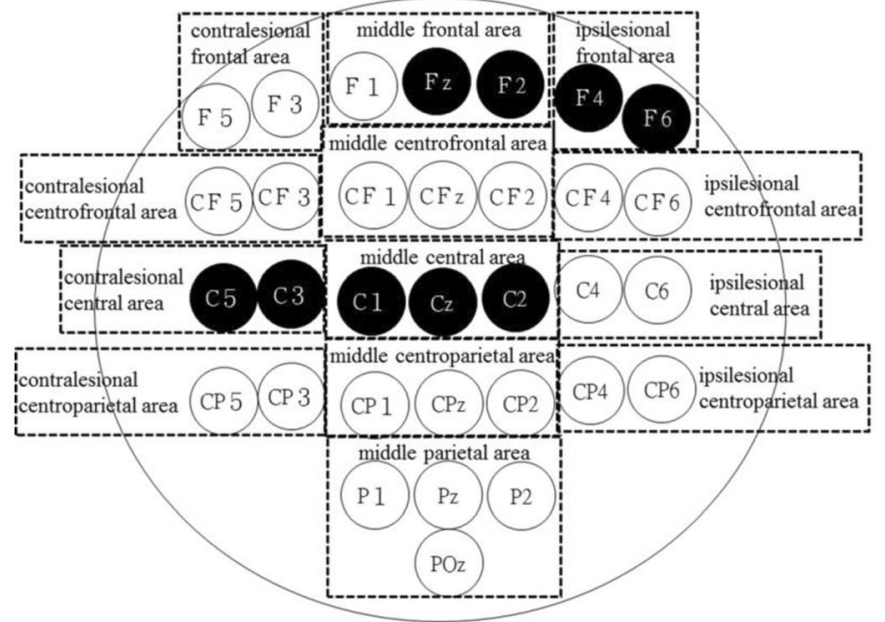

Fig. 3 (a) Functional EEG-EMG connectivity in the gamma band. The signals were recorded during regular treadmill walking. The black circles represent the corticomuscular connectivity with significant intergroup difference. (b) The means and standard deviations of the EEG-EMG connectivity values are shown for the regions with significant intergroup differences. ${ }^{*}$ denotes a significance level $<0.05$ for intergroup comparisons (control vs. experimental). (c) Changes in the functional EEG-EMG connectivity in the gamma band correlate with the recovery of temporal gait asymmetry. The black circles represent the changes in the EEG-EMG pairs with significant intergroup differences that were negative correlated with the changes in the temporal asymmetry ratio. For better visualization and interpretation, the left represents the contralesional hemisphere, and the right represents the ipsilesional hemisphere 
A



B

B $0.8 \quad$ Alpha band

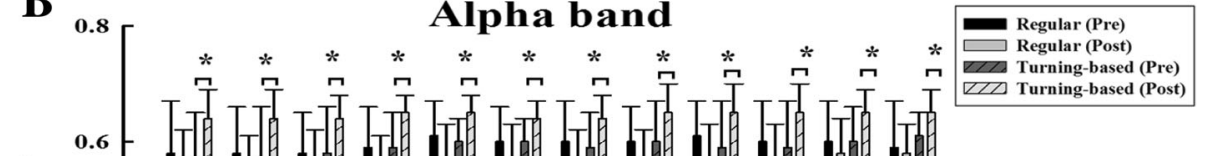

를

0.4

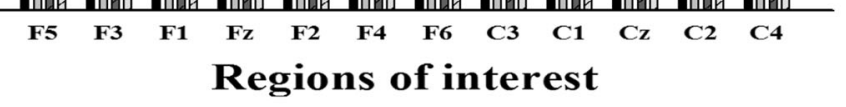

C

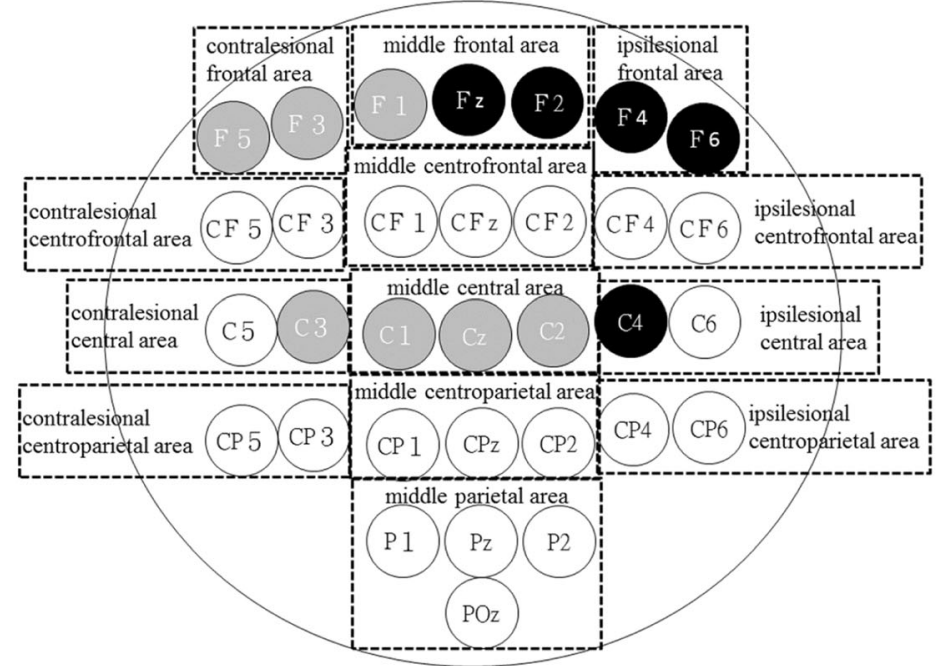

Fig. 4 (See legend on next page.) 
(See figure on previous page.)

Fig. 4 (a) Functional EEG-EMG connectivity in the alpha band. The signals were recorded during regular treadmill walking. The black circles represent the corticomuscular connectivity with significant intergroup differences. (b) The means and standard deviations of the EEG-EMG connectivity values are shown for the regions with significant intergroup differences. * denotes a significance level $<0.05$ for intergroup comparisons (control vs. experimental). (c) Changes in the functional EEG-EMG connectivity in the alpha band correlate with the recovery of temporal gait asymmetry. The black circles represent the changes in the EEG-EMG pairs with significant intergroup differences that negatively correlate to the changes in the temporal asymmetry ratio. The gray circles represent the changes in the EEG-EMG pairs with significant intergroup differences that do not correlate to the changes in the temporal asymmetry ratio. For better visualization and interpretation, the left represents the contralesional hemisphere, and the right represents the ipsilesional hemisphere

was primarily due to a reduction in the single support time on the unaffected leg. Therefore, our turning-based treadmill training seems to have a stronger effect on advancing the affected leg than regular treadmill training. In addition, temporal gait symmetry is an important gait parameter that provides information on energy consumption [19, 28]. The gait asymmetry was not improved after 12 sessions of regular treadmill training, which may have be due to task repetition reinforcement of asymmetry on the treadmill [29]. However, the gait asymmetry was improved after turning-based treadmill training in the present study. We thus suggest that this novel training can be applied to improve affected leg advancement and possibly reduce energy consumption in stroke rehabilitation.

In this study, we further demonstrated the improvements in walking speed and gait symmetry accompanied by concurrent modulation in EEG-EEG and EEG-EMG connectivity. Moreover, the larger the changes in functional connectivity were, either in EEG-EEG or EEG-EMG connectivity, the better the recovery of gait symmetry. Therefore, such neuromuscular modulation or neural plasticity induced by training may, at least partially, explain the gait improvement. A previous study suggested that individuals with chronic stroke preserve the capacity to increase synchronization of neural activity between different brain regions as measured by EEG connectivity through neurofeedback training [1]. It has been noted that a challenging, unfamiliar and more difficult task for stroke patients may increase the possibilities for brain activation to stimulate behavioral recovery [3]. Turning-based walking requires more balance and limb coordination than walking in straight line [30]. Therefore, walking on a turning-based treadmill seems to fulfill the features necessary for a task to induce brain activation and thus behavior recovery.

In this study, the EEG-EEG connectivity and EEG-EMG connectivity measured during treadmill walking was used to explore the possible mechanisms for reorganization of functional cortical network and functional coupling between cortical commands and subsequent muscle activation. Involvement of the motor cortex and corticospinal tract in the control of walking has been demonstrated in healthy subjects $[5,8]$. However, how novel gait training shapes brain activities and the descending pathway for chronic stroke survivors remains unclear. We revealed both cortico-cortical and cortico-muscular reorganization in the gamma band over the middle central area, middle frontal area, ipsilesional frontal, and contralesional central area can be further enhanced by challenging gait training, such as the turning-based treadmill training in this study. One possible role of coherent oscillation is to link and promote synchronous neural firing between different neuronal populations with different spatial distributions but that are functionally related [31]. The coupling between EEG and EMG indicated that cortical control drives peripheral muscular activities through the corticospinal tract during walking. Increased connectivity in the gamma band after specific walking training is in line with previous results that showed that the peak EEG-EMG coherence frequency always shifted to higher frequency $(25-40 \mathrm{~Hz})$ from the beta-band during walking compared to those during static contraction [8]. The gamma-band oscillations in the frontal-central areas play an important role in the execution of the complex goal-directed task which involved motor coordination, cognitive processes and sensorimotor integration [32]. Therefore, the turning-based

Table 3 Gait performance

\begin{tabular}{|c|c|c|c|c|c|c|}
\hline \multirow[b]{2}{*}{ Measures } & \multicolumn{2}{|c|}{ Control group $(n=9)$} & \multicolumn{2}{|c|}{ Experimental group $(n=9)$} & \multirow{2}{*}{$\begin{array}{l}\text { Time } \\
\text { Effect, } \\
P\end{array}$} & \multirow{2}{*}{$\begin{array}{l}\text { Time } \times \\
\text { Group, } \\
P\end{array}$} \\
\hline & Pre & Post & Pre & Post & & \\
\hline Speed $(\mathrm{m} / \mathrm{s})$ & $0.64 \pm 0.30$ & $0.66 \pm 0.31$ & $0.58 \pm 0.23$ & $0.71 \pm 0.31^{*}$ & 0.01 & 0.037 \\
\hline Temporal asymmetry ratio & $0.29 \pm 0.16$ & $0.31 \pm 0.15$ & $0.30 \pm 0.12$ & $0.24 \pm 0.08^{*}$ & 0.138 & 0.026 \\
\hline
\end{tabular}

Values are the mean \pm standard deviation

*Significance level < 0.05 for intergroup comparisons (control vs. experimental) 
Table 4 Individual data of gait performance

\begin{tabular}{|c|c|c|c|c|c|}
\hline & \multirow[b]{2}{*}{ Subject number } & \multicolumn{2}{|l|}{ Speed $(\mathrm{m} / \mathrm{s})$} & \multicolumn{2}{|c|}{ Temporal asymmetry ratio } \\
\hline & & Pre & Post & Pre & Post \\
\hline \multirow[t]{10}{*}{ Control group } & 1 & 1.00 & 1.02 & 0.27 & 0.28 \\
\hline & 2 & 0.82 & 0.75 & 0.09 & 0.1 \\
\hline & 3 & 0.78 & 0.98 & 0.12 & 0.11 \\
\hline & 4 & 0.83 & 0.73 & 0.40 & 0.35 \\
\hline & 5 & 0.21 & 0.29 & 0.57 & 0.46 \\
\hline & 6 & 0.26 & 0.19 & 0.24 & 0.42 \\
\hline & 7 & 0.59 & 0.67 & 0.26 & 0.22 \\
\hline & 8 & 0.88 & 0.93 & 0.21 & 0.30 \\
\hline & 9 & 0.38 & 0.36 & 0.48 & 0.52 \\
\hline & Mean (SD) & $0.64(0.30)$ & $0.66(0.31)$ & $0.29(0.16)$ & $0.31(0.15)$ \\
\hline \multirow[t]{10}{*}{ Experimental group } & 1 & 0.75 & 0.99 & 0.12 & 0.10 \\
\hline & 2 & 0.89 & 1.00 & 0.17 & 0.18 \\
\hline & 3 & 0.74 & 1.02 & 0.26 & 0.16 \\
\hline & 4 & 0.52 & 0.75 & 0.27 & 0.23 \\
\hline & 5 & 0.47 & 0.63 & 0.41 & 0.28 \\
\hline & 6 & 0.68 & 0.92 & 0.42 & 0.32 \\
\hline & 7 & 0.26 & 0.26 & 0.46 & 0.37 \\
\hline & 8 & 0.68 & 0.66 & 0.22 & 0.22 \\
\hline & 9 & 0.23 & 0.19 & 0.39 & 0.26 \\
\hline & Mean (SD) & $0.58(0.23)$ & $0.71(0.31)$ & $0.30(0.12)$ & $0.24(0.08)$ \\
\hline
\end{tabular}

treadmill training, which includes specific training and requires complex integration of coordinated muscle activity and multiple sensory systems by the cortex, could result in better walking performance.

Assessment of EEG functional connectivity has been widely used to understand the correlation of brain activities in different cortical areas [14]. The coherent neural oscillation in the beta band over the ipsilesional motor cortex was able to predict motor improvements in the first week after stroke [33]. Loss of connectivity thus suggests a low capacity to integrate sensorimotor communication between distant brain regions in stroke survivors [34]. Following 28 days of upper-extremity treatment increased the resting-state connectivity of the beta-band between the ipsilesional primary motor cortex and premotor cortex paralleling the motor gains [34]. A recent study showed that 40-session gait training with powered exoskeletons could improve the strength of functional connectivity in the affected hemisphere [35]. Consistent with above results, we provide evidence that this 12-session turning-based treadmill training for chronic stroke patients could induce synchronization of the cortical network around distinct brain areas together with gait improvement. Furthermore, this challenging turning-based training was demonstrated to be more effective than regular treadmill training with regard to brain modulation and gait performance.

Besides the involvement of neural relocation in the ipsilesional hemisphere, we observed the network contribution over the contralesional frontal-central-parietal area and between the contralesional central area and the lower-extremity muscle for gait recovery. The role of the contralesional motor area for treatment-induced poststroke brain reorganization is controversial. Decreased frontoparietal networking in the contralesional hemisphere has only been found in stroke patients who gained improved motor behavior after ankle robotics training [36]. However, as some recent studies emphasized the role of the contralesional hemisphere during recover [34], our results showed that activation in the contralesional hemisphere did not necessarily contribute to unfavorable recovery, especially for chronic stroke survivors. In addition, motor task selection and duration of training session may also account for such discrepancies.

In addition to the intrahemispheric interactions, increased interhemispheric connectivity after turning-based treadmill training was observed. To our knowledge, evidence of the interhemispheric connectivity induced by lower-extremity training is nonexistent. However, these findings were consistent with previous studies' findings of the strong influence of the ipsilesional dorsal premotor 
cortex on its contralesional homologue, with improvements in behavioral performance after 3 weeks of upper limb rehabilitation therapy in stroke patients [37]. Pellegrino et al. [38] also demonstrated that the 12-week robotic treatment for chronic stroke patients improved hand control and modulated the interhemispheric connectivity in the high beta and gamma bands at rest and such changes correlated with hand control improvements. We reported the first evidence that interhemispheric connectivity during walking can be shaped by novel treadmill training. Considering the bilateral involvement of the lower-extremities during walking, neurophysiological mechanisms of walking regarding the interaction between the lesioned and contralesioned hemispheres in stroke patients warrants further study.

Interestingly, increased coherence in the alpha band was only present in the EEG-EMG connectivity in this study. It is well known that the coherence in the 8-12 $\mathrm{Hz}$ frequency over the motor area is most prominent during rest, and such coherence is suppressed (or called desynchronization) during voluntary movement [39]. However, a recent study found the coherence between EEG and EMG of TA around this frequency band could be induced by peripheral nerve stimulation while performing static muscle contractions [40]. Petersen et al. also reported a coherence peak at approximately $10 \mathrm{~Hz}$ during walking in healthy human subjects [8]. Our present findings may reflect the possibility of treatment-induced reorganization from afferent inputs to the cortical network during walking [2].

It is noteworthy that enhancements in gait symmetry, but not the gait speed, were related to the modulations in EEG-EEG and EEG-EMG connectivity over the frontal-central-parietal areas in the brain. The network changes may thus account for the associated gait recovery underlying the central and peripheral neuromuscular mechanisms. The temporal gait symmetry provides information on the differences of single support times between the 2 legs [20]. As mentioned above, improvement of temporal gait symmetry may be explained by the successful combination of a forced compelled weight-bearing approach and intensive practice of the normal swing phase component. The control of a normal gait pattern demands that patients achieve more muscular control and whole-body coordination [19]. Therefore, the restoration of gait pattern, rather than gait speed, is more likely to relate to the alteration of brain activities. Although speed is the most widely used measurement and can differentiate levels of gait disability, it is determined by multiple factors [20]. Investigation of cortical contribution for gait speed in response to therapeutic approaches is needed in the future. Recently, numerous studies have started to design the
EEG-based brain-machine interfaces (BMI) to decode the association between the angles of specific joints and neuroelectric cortical activity during gait training $[41,42]$. Since gait symmetry assists in understanding the underlying impairments and treatment-induced cortical changes, a measure of temporal gait symmetry should be included in quantitative gait analysis to better reflect this aspect in gait training with an EEG-based BMI.

This study has several limitations. The sample size is relatively small, and we only assessed limited gait parameters. A larger, randomized controlled clinical trial including comprehensive assessment of gait parameters (i.e., distinct aspects of symmetry or accelerometry signals in time, frequency and time-frequency domains [43]) is needed to validate the reported brain characteristics of the treadmill training in this study. The improved gait speed and temporal symmetry maintained at 1-month follow-up according to our previous works [2], however, whether the brain network-related gait recovery can be seen at follow-up is not known. Additionally, only ambulatory patients with chronic stroke were recruited. Therefore, our results may not extend to individuals with acute stroke or more severe motor deficits. In this study, we only provided 32-channel cortical data and one muscle signal to explore possible connectivity during walking. Future studies should use a combination of anatomical and functional neuroimaging techniques to determine the spatial patterns of brain reorganization after gait training. In addition, the task chosen for neurophysiological assessment was walking on the regular treadmill for both groups. Therefore, the assessed task might be more familiar to participants in the regular treadmill training group, but not to participants in the turning-based training group. Whether neural connectivity is influenced by task familiarity is not clear and needs further elucidation. The lack of a complete understanding of the cortical contribution to walking ability and walking recovery after a stroke is due to lacking of valid tools to thoroughly investigate the functional role of the cortex during walking in humans. As mentioned in the methods section, although we have done our best to exclude all motion artifacts and provide stable task-related EEG signals, we believe future studies using more sophisticated engineering solutions for eliciting artifacts could advance our understanding of the "real-time" brain activities during walking and boost development of effective intervention with a neural basis $[44,45]$.

\section{Conclusions}

Despite thorough documentation of the unique neural correlations during motor function, previous EEG studies have rarely focused on changes in brain activity during 
walking in people with stroke after neurorehabilitation. In the present study, we were the first to apply TFCMI, a nonlinear, noise-resistant method within task-related frequency, to explore the EEG-EEG/EEG-EMG connectivity for treatment-induced brain reorganization after a specific gait training program. Our results demonstrated that 12 sessions of this 30-min novel gait training on a turningbased treadmill improved network-related gait recovery with underlying cortico-cortical and corticomuscular mechanisms.

\section{Abbreviations}

BMI: Brain-machine interfaces; CC: Contralesional central area;

CCP: Contralesional central-parietal area; CF: Contralesional frontal area;

CFC: Contralesional frontal-central area; HEOG: Horizontal electrooculography;

IC: Ipsilesional central area; ICA: Independent component analysis;

ICP: Ipsilesional central-parietal area; IF: Ipsilesional frontal area;

IFC: Ipsilesional frontal-central area; MC: Middle central area; MCP: Middle

central-parietal area; MF: Middle frontal area; MFC: Middle frontal-central area; MMSE: Mini-mental state examination; MP: Middle parietal area; pdf: Probability density function; TA: Tibialis anterior; TFCMI: Time-frequency cross mutual information; VEOG: Vertical electrooculography

\section{Acknowledgements}

The authors would like to thank the study participants.

\section{Funding}

Support for this study was provided by the National Health Research Institutes of the Republic of China (Grant No. NHRIEX100-10039EI, NHRIEX101-10039EI) and the Ministry of Science and Technology of the Republic of China (Grant No. MOST-103-2314-B-010-002-MY3). The funding body had no role in the study design, data collection, analysis, and interpretation, or preparation of the manuscript.

\section{Availability of data and materials}

The datasets used and/or analyzed for this study are available from the corresponding author on reasonable request.

\section{Authors' contributions}

IHC conducted the experiment, analyzed the results and wrote the manuscript; YRY conceived the experiment; CFL wrote the analysis script; and RYW conceived the experiment and reviewed the manuscript. All authors read and approved the final manuscript.

\section{Ethics approval and consent to participate}

The study protocol was approved by the institutional review board of Taipei City Hospital. Participants consented to participate following an explanation of the procedure and review of the informed consent.

\section{Consent for publication}

Not applicable.

\section{Competing interests}

The authors declare that they have no competing interests.

\section{Publisher's Note}

Springer Nature remains neutral with regard to jurisdictional claims in published maps and institutional affiliations.

\section{Author details}

${ }^{1}$ Department of Physical Therapy, Fooyin University, Kaohsiung, Taiwan. ${ }^{2}$ Department of Physical Therapy and Assistive Technology, National Yang-Ming University, 155, Sec 2, Li Nong St., Shih-Pai, Taipei 112, Taiwan. ${ }^{3}$ Department of Biomedical Imaging and Radiological Sciences, National Yang-Ming University, Taipei, Taiwan.
Received: 2 October 2017 Accepted: 22 February 2019

Published online: 28 February 2019

\section{References}

1. Mottaz A, Corbet T, Doganci N, Magnin C, Nicolo P, Schnider A, Guggisberg AG. Modulating functional connectivity after stroke with neurofeedback: effect on motor deficits in a controlled cross-over study. Neuroimage Clin. 2018;20:336-46.

2. Chen $\mathrm{H}$, Yang YR, Chan RC, Wang RY. Turning-based treadmill training improves turning performance and gait symmetry after stroke. Neurorehabil Neural Repair. 2014:28:45-55.

3. Krakauer JW. Motor learning: its relevance to stroke recovery and neurorehabilitation. Curr Opin Neurol. 2006;19:84-90.

4. Gwin JT, Gramann K, Makeig S, Ferris DP. Electrocortical activity is coupled to gait cycle phase during treadmill walking. Neuroimage. 2011;54:1289-96.

5. Knaepen K, Mierau A, Tellez HF, Lefeber D, Meeusen R. Temporal and spatial organization of gait-related electrocortical potentials. Neurosci Lett. 2015;599:75-80.

6. Grefkes C, Fink GR. Reorganization of cerebral networks after stroke: new insights from neuroimaging with connectivity approaches. Brain. 2011;134:1264-76.

7. von Carlowitz-Ghori K, Bayraktaroglu Z, Hohlefeld FU, Losch F, Curio G, Nikulin W. Corticomuscular coherence in acute and chronic stroke. Clin Neurophysiol. 2014;125:1182-91.

8. Petersen TH, Willerslev-Olsen M, Conway BA, Nielsen JB. The motor cortex drives the muscles during walking in human subjects. J Physiol. 2012;590: 2443-52.

9. Youssofzadeh V, Zanotto D, Wong-Lin K, Agrawal S, Prasad G. Directed functional connectivity in Fronto-Centroparietal circuit correlates with motor adaptation in gait training. IEEE Trans Neural Syst Rehabil Eng. 2016;24: $1265-75$.

10. Pohl M, Mehrholz J, Ritschel C, Ruckriem S. Speed-dependent treadmill training in ambulatory Hemiparetic stroke patients: a randomized controlled trial. Stroke. 2002;33:553-8.

11. Popivanov D, Dushanova J. Non-linear EEG dynamic changes and their probable relation to voluntary movement organization. Neuroreport. 1999; 10:1397-401.

12. Nunez PL, Srinivasan R, Westdorp AF, Wijesinghe RS, Tucker DM, Silberstein RB, Cadusch PJ, Coherency EEG. I: Statistics, reference electrode, volume conduction, Laplacians, cortical imaging, and interpretation at multiple scales Electroencephalogr. Clin Neurophysiol. 1997:103:499-515.

13. Andrew C, Pfurtscheller G. Lack of bilateral coherence of post-movement central beta oscillations in the human electroencephalogram. Neurosci Lett. 1999;273:89-92.

14. Lu CF, Teng $\mathrm{S}$, Hung $\mathrm{Cl}$, Tseng PJ, Lin LT, Lee PL, Wu YT. Reorganization of functional connectivity during the motor task using EEG time-frequency cross mutual information analysis. Clin Neurophysiol. 2011;122:1569-79.

15. Chen CC, Hsieh JC, Wu YZ, Lee PL, Chen SS, Niddam DM, Yeh TC, Wu YT. Mutual-information-based approach for neural connectivity during selfpaced finger lifting task. Hum Brain Mapp. 2008;29:265-80.

16. Mima T, Toma K, Koshy B, Hallett M. Coherence between cortical and muscular activities after subcortical stroke. Stroke. 2001;32:2597-601.

17. Hermens HJ, Freriks B, Disselhorst-Klug C, Rau G. Development of recommendations for SEMG sensors and sensor placement procedures. J Electromyogr Kinesiol. 2000:10:361-74.

18. Vialatte FB, Martin C, Dubois R, Haddad J, Quenet B, Gervais R, Dreyfus G. A machine learning approach to the analysis of time-frequency maps, and its application to neural dynamics. Neural Netw. 2007;20:194-209.

19. Silver KH, Macko RF, Forrester LW, Goldberg AP, Smith GV. Effects of aerobic treadmill training on gait velocity, cadence, and gait symmetry in chronic hemiparetic stroke: a preliminary report. Neurorehabil Neural Repair. 2000;14:65-71.

20. Patterson KK, Gage WH, Brooks D, Black SE, Mcllroy WE. Changes in gait symmetry and velocity after stroke: a cross-sectional study from weeks to years after stroke. Neurorehabil Neural Repair. 2010;24:783-90.

21. Peters DM, Middleton A, Donley JW, Blanck EL, Fritz SL. Concurrent validity of walking speed values calculated via the GAITRite electronic walkway and 3 meter walk test in the chronic stroke population. Physiother Theory Pract. 2014;30:183-8. 
22. Lewek MD, Randall EP. Reliability of spatiotemporal asymmetry during overground walking for individuals following chronic stroke. J Neurol Phys Ther. 2011;35:116-21.

23. Patterson KK, Parafianowicz I, Danells CJ, Closson V, Verrier MC, Staines WR, Black SE, Mcllroy WE. Gait asymmetry in community-ambulating stroke survivors. Arch Phys Med Rehabil. 2008:89:304-10.

24. Helm EE, Reisman DS. The Split-Belt walking paradigm: Exploring Motor Learning and Spatiotemporal Asymmetry Poststroke. Phys Med Rehabil Clin N Am. 2015;26:703-13

25. Daly JJ, Ruff RL. Construction of efficacious gait and upper limb functional interventions based on brain plasticity evidence and model-based measures for stroke patients. ScientificWorldJournal. 2007;7:2031-45.

26. Courtine G, Schieppati M. Human walking along a curved path. II. Gait features and EMG patterns. Eur J Neurosci. 2003;18:191-205.

27. Chen IH, Yang YR, Cheng SJ, Chan RC, Wang RY. Neuromuscular and biomechanical strategies of turning in ambulatory individuals post-stroke. Chin J Physiol. 2014;57:128-36.

28. Kim CM, Eng JJ. Symmetry in vertical ground reaction force is accompanied by symmetry in temporal but not distance variables of gait in persons with stroke. Gait Posture. 2003;18:23-8.

29. Patterson SL, Rodgers MM, Macko RF, Forrester LW. Effect of treadmill exercise training on spatial and temporal gait parameters in subjects with chronic stroke: a preliminary report. J Rehabil Res Dev. 2008:45:221-8.

30. Courtine G, Papaxanthis C, Schieppati M. Coordinated modulation of locomotor muscle synergies constructs straight-ahead and curvilinear walking in humans. Exp Brain Res. 2006;170:320-35.

31. Marsden JF, Ashby P, Limousin-Dowsey P, Rothwell JC, Brown P. Coherence between cerebellar thalamus, cortex and muscle in man: cerebellar thalamus interactions. Brain. 2000;123:1459-70.

32. Teixeira S, Velasques B, Machado S, Cunha M, Domingues CA, Budde $H$, Anghinah R, Basile LF, Cagy M, Piedade R, Ribeiro P. Gamma-band oscillations in fronto-central areas during performance of a sensorimotor integration task: a qEEG coherence study. Neurosci Lett. 2010;483:114-7.

33. Nicolo P, Rizk S, Magnin C, Pietro MD, Schnider A, Guggisberg AG. Coherent neural oscillations predict future motor and language improvement after stroke. Brain. 2015;138:3048-60.

34. Wu J, Quinlan EB, Dodakian L, McKenzie A, Kathuria N, Zhou RJ, Augsburger R, See J, Le VH, Srinivasan R, Cramer SC. Connectivity measures are robust biomarkers of cortical function and plasticity after stroke. Brain. 2015;138:2359-69.

35. Calabro RS, Naro A, Russo M, Bramanti P, Carioti L, Balletta T, Buda A, Manuli A, Filoni S, Bramanti A. Shaping neuroplasticity by using powered exoskeletons in patients with stroke: a randomized clinical trial. J Neuroeng Rehabil. 2018;15:35.

36. Goodman RN, Rietschel JC, Roy A, Jung BC, Diaz J, Macko RF, Forrester LW Increased reward in ankle robotics training enhances motor control and cortical efficiency in stroke. J Rehabil Res Dev. 2014;51:213-27.

37. James GA, Lu ZL, VanMeter JW, Sathian K, Hu XP, Butler AJ. Changes in resting state effective connectivity in the motor network following rehabilitation of upper extremity poststroke paresis. Top Stroke Rehabil. 2009;16:270-81.

38. Pellegrino G, Tomasevic L, Tombini M, Assenza G, Bravi M, Sterzi S, Giacobbe V, Zollo L, Guglielmelli E, Cavallo G, et al. Inter-hemispheric coupling changes associate with motor improvements after robotic stroke rehabilitation. Restor Neurol Neurosci. 2012;30:497-510.

39. van der Helden J, van Schie HT, Rombouts C. Observational learning of new movement sequences is reflected in fronto-parietal coherence. PLoS One. 2010;5:e14482

40. Hansen NL, Nielsen JB. The effect of transcranial magnetic stimulation and peripheral nerve stimulation on corticomuscular coherence in humans. J Physiol. 2004;561:295-306.

41. Contreras-Vidal JL, Bortole M, Zhu F, Nathan K, Venkatakrishnan A, Francisco GE, Soto R, Pons JL. Neural decoding of robot-assisted gait during rehabilitation after stroke. Am J Phys Med Rehabil. 2018;97:541-50

42. Youssofzadeh V, Zanotto D, Stegall P, Naeem M, Wong-Lin K, Agrawal SK, Prasad G. Directed neural connectivity changes in robot-assisted gait training: a partial granger causality analysis. Conf Proc IEEE Eng Med Biol Soc. 2014;2014:6361-4

43. Sejdic E, Lowry KA, Bellanca J, Redfern MS, Brach JS. A comprehensive assessment of gait accelerometry signals in time, frequency and timefrequency domains. IEEE Trans Neural Syst Rehabil Eng. 2014;22:603-12.
44. Oliveira AS, Schlink BR, Hairston WD, Konig P, Ferris DP. Induction and separation of motion artifacts in EEG data using a mobile phantom head device. J Neural Eng. 2016;13:036014.

45. Symeonidou ER, Nordin AD, Hairston WD, Ferris DP. Effects of cable sway, electrode surface area, and electrode mass on electroencephalography signal quality during motion. Sensors (Basel). 2018;18.

\section{Ready to submit your research? Choose BMC and benefit from:}

- fast, convenient online submission

- thorough peer review by experienced researchers in your field

- rapid publication on acceptance

- support for research data, including large and complex data types

- gold Open Access which fosters wider collaboration and increased citations

- maximum visibility for your research: over $100 \mathrm{M}$ website views per year

At BMC, research is always in progress.

Learn more biomedcentral.com/submissions 